\title{
A Review of Surgical Aortic Valve Repair. Indications and techniques for the General Cardiologist
}

\section{Jeremy W. Docekal,}

Landstuhl Regional Military Medical Center Department of Cardiology

Corresponding author: Jeremy William Docekal, Staff cardiologist Landstuhl Regional Military medical Center.

Received date: July 09, 2020; Accepted date: July 14, 2020; Published date: July 29, 2020

Citation: Jeremy W. Docekal., (2020) A Review of Surgical Aortic Valve Repair. Indications and techniques for the General Cardiologist.

J,Clinical Cardiology and Cardiovascular Interventions, 3(7); Doi:10.31579/2641-0419/073

Copyright: @ 2020 Jeremy William Docekal, This is an open access article distributed under the Creative Commons Attribution License, which permits unrestricted use, distribution, and reproduction in any medium, provided the original work is properly cited.

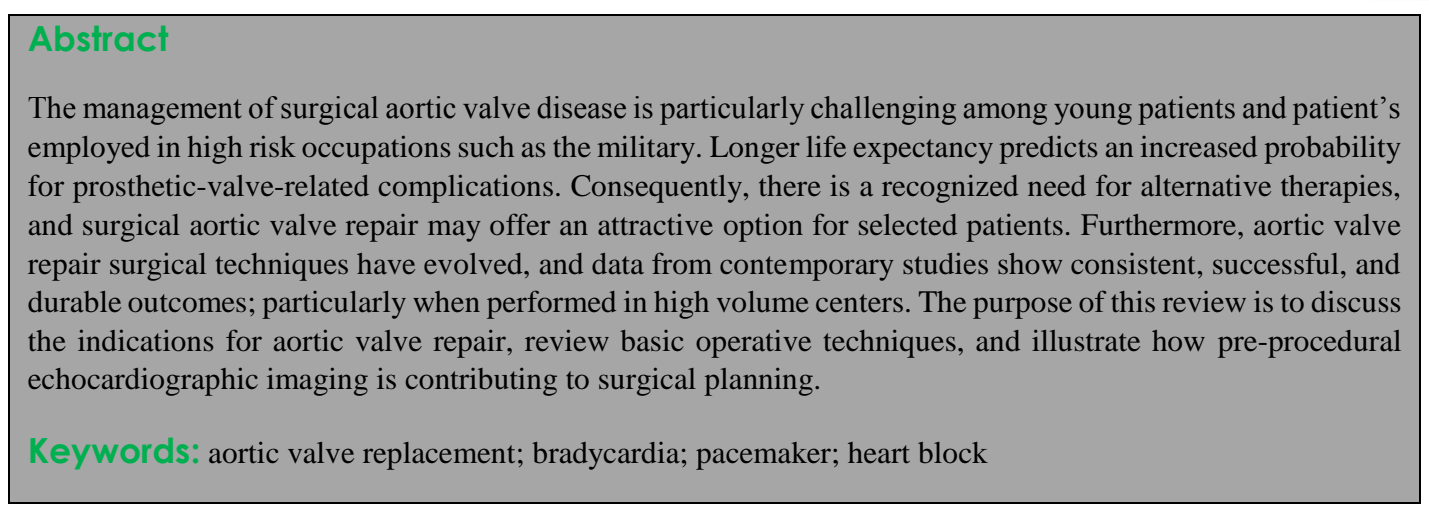

\section{Introduction}

Young patients, particularly those employed in high-risk occupations such as the military or among competitive athletes, who have aortic valve pathology, present a well-documented challenge in the current era. For these patients, postoperative quality of life, occupational compatibility, and life expectancy are important considerations affecting choice of therapy. A commonly offered solution is aortic valve replacement (AVR) with either a mechanical or biologic prosthesis. However, AVR is associated with several disadvantages.
In a recently published study on this topic, the authors conclude that their study found a "shorter life expectancy in patients after aortic valve replacement (AVR) compared with the general population. The estimated loss in life expectancy was substantial, and increased with younger age (Figure 1) [1]." Their observation may be explained, in part, by the fact that longer life expectancy predicts increased exposure to prostheticvalve-related complications such as degeneration leading to reoperation, bleeding, and thromboembolism [2].
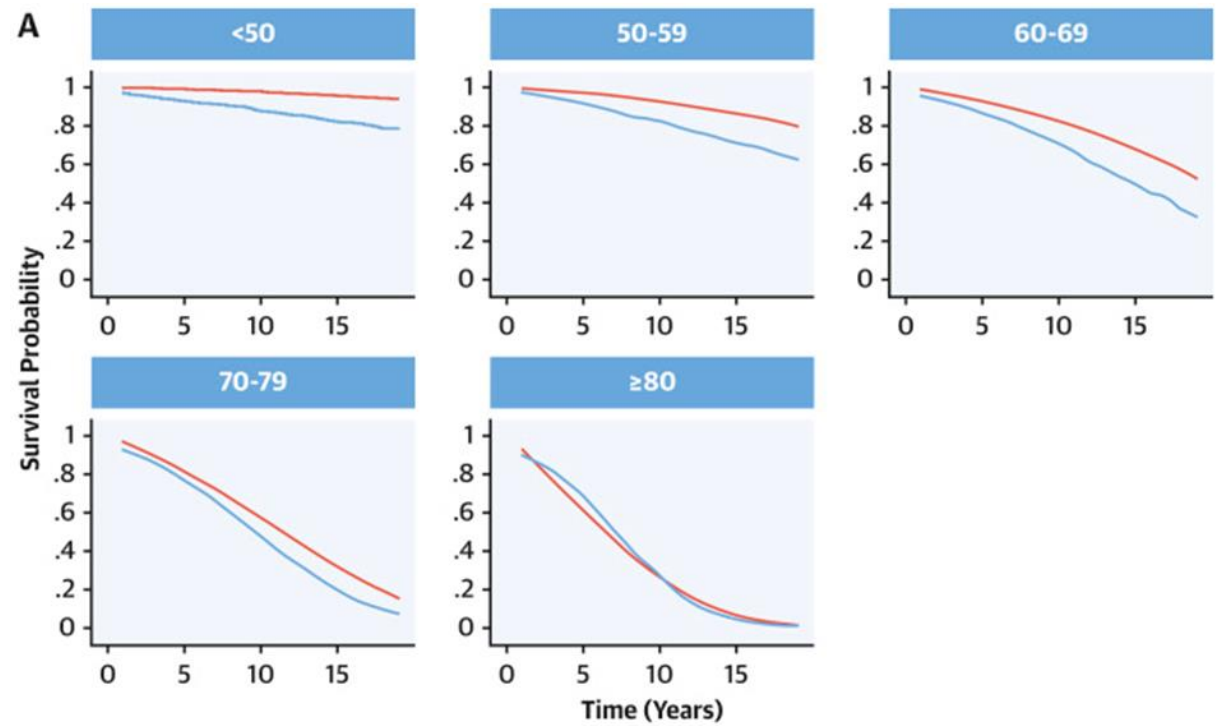
Figure 1: The observed survival in patients after aortic valve replacement (blue line) compared with the expected survival of an age-, sex-, and calendaryear-matched Swedish population (red line). (From Glaser N, Persson M, Jackson V, Holzmann M, Franco-Cereceda A, Sartipy U. Loss in life expectancy after surgical aortic valve replacement. J Am Coll Cardiol 2019; 74:26-33).

A retrospective review by Goldstone et al. [3] helps us to better understand the magnitude of the problem. Their study observed, among patients aged $45-54$, a 15 -year mortality rate of $30.6 \%$ in patients who received AVR with a biologic prosthesis and an estimated 15-year mortality rate of $26.4 \%$ following AVR with a mechanical prosthesis. Furthermore, a retrospective review performed in Ottawa, Canada concluded that the median interval to reoperation for contemporary stented aortic bioprosthesis was 7.74 years (95\% CI 7.28 to 9.97 years,) in patients younger than 40 years of age [4].

With the disconcerting data emerging in regard to the long-term benefit of AVR, there is a need for alternative options. For this reason, there is a renewed interest in two surgical options in particular. Among young patients with aortic valve disease, the Ross procedure has several demonstrated advantages. Buratto et al. recently demonstrated that the Ross procedure is associated with a reduced risk of late mortality as compared with mechanical AVR, which may be partially explained by avoidance of anticoagulation and its associated complications. Furthermore, the Ross procedure achieves more favorable valve hemodynamics, whereby the effective orifice area is greater than that which can be achieved with a prosthesis [5]. The advantages of the Ross procedure have been recently and elegantly reviewed, and this is not the primary intent of this paper [6]. A second option is surgical aortic valve repair (AV repair), which is has long been regarded as a surgical alternative to prosthetic valve replacement among selected patients with aortic insufficiency (AI) or aortic aneurysm.

To help illustrate the advantage of AV repair, in a propensity score analysis matching patients who underwent surgical correction of severe AI by either surgical AVR or AV repair, it was determined that AV repair is associated with a better overall 9-year survival rate as compared to AVR (87\% vs $60 \%$; $\mathrm{P}=.007$ ) [7]. In addition, a report published by the Society of Thoracic Surgeons confirms a low operative mortality among patients who underwent aortic valve sparing procedures (1.88\%), which compares favorably with reported mortality rates of $5 \%$ among surgical AVR using a valved conduit (Bentall procedure) [8].

Yet, in spite of the published advantages of AV repair, this remains an underutilized surgical technique, particularly in the United States. In a report from the Society of Thoracic Surgeons database [8], it was determined that there is an increasing trend towards AVR using biostented valves, while AV repair remains an uncommon procedure for most surgical centers. According to this same report, currently only 5\% of institutions participating in the Society of Thoracic Surgeons Adult Cardiac Surgery database (STS ACSD) performed >16 aortic valve sparing procedures in 2009.

Given the proposed advantages of AV repair and increasing familiarity of this procedure in several European institutions, it remains difficult to justify slow adoption of AV repair in the United States. One possible explanation is the historic wide range in reported surgical outcomes with AV repair. However, contemporary publications of AV repair results from specialized centers are now available and demonstrate excellent short- and long-term results. In addition, an industry generated bias towards valve replacement with current mechanical and bioprosthetic implants may possibly exist.

Advances in echocardiographic imaging have helped improve surgical planning. Thus, the cardiologist with training in 3-dimensional echocardiography now provides valuable insight and is an active contributor to successful AV repair.

The purpose of this review article is to discuss the indications for AV repair, discuss candidate patients, review current outcomes data, and help familiarize the reader in regard to basic surgical techniques used for AV repair. In addition, we review how advanced, non-invasive imaging is currently helping improve surgical planning and outcomes.

\section{Anatomy of the aortic root}

Prior to further discussion, a review of aortic root anatomy is instructive.

In general, the aortic root may be thought of as a bridge between the left ventricle and the ascending aorta, by which the aortic root acts as a native stent, surrounding and supporting the 3 aortic cusps (11).

In terms of function, valve integrity depends upon the cohesion of all structures forming the aortic root: the aortic valve leaflets and their attachments, Sinuses of Valsalva (SoV), interleaflet trigones, sinotubular junction (STJ), and the aortic annulus.

The aortic leaflets form the hemodynamic junction between the left ventricle and aorta and form the demarcation between structures subject to arterial pressures and those subjected to ventricular pressures $[9,10]$. The aortic leaflets are attached to the aortic wall in a semilunar fashion and extend basally from the ventriculo-aortic junction (VAJ) to their distal attachment at the STJ. As the leaflet attachments insert into the wall of the aorta, they form a crown-shaped fibrous structure [11].

The anatomy of the valve leaflets is composed of the load bearing leaflet body, the free margin (otherwise known as the coapting surface) that contains a thickened circular node termed the nodule of Arantius [9].), and the leaflet attachment (otherwise known as the basal leaflet or hinge point) [9]. The free edge of the aortic leaflets are constructed such that when closed, the leaflets coapt over several millimeters. The margin of overlap has been defined as the lunula [12]. The term commissure refers to the zone of apposition between the two coronet-shaped, lateral attachments of adjacent leaflets to the aortic wall. A failure in the development of the commissural area results in the development of bicuspid aortic valves (BAV), as explained later in this text. The term "cusp" is used to describe the leaflet tip, but is commonly used synonymously with the term leaflet.

The exact definition of the aortic annulus is debated, and there is an absence of universally agreed upon criteria from either an anatomic or hemodynamic viewpoint. However, there exists a condensation of collagenous tissue at the leaflet hinge point, which follows the semi-lunar contour of the valvular attachment and is thickest at the nadir of the 
semilunar attachments [12]. This ring of collagenous tissue provides the substrate which anatomically defines the annulus. However, the functional annulus determines valve geometry and provides an important anatomical landmark for surgical repair. The functional annulus is determined by a horizontal plane connecting the cusp nadirs and is termed the basal ring (See Figure 2). This is discussed in more detail later in this text. The near universal desire to describe the structure of the aortic valve in terms of annulus has perhaps superseded the key fact that it is the semilunar arrangement of leaflet hinge points which is critical to normal valvular function [13].

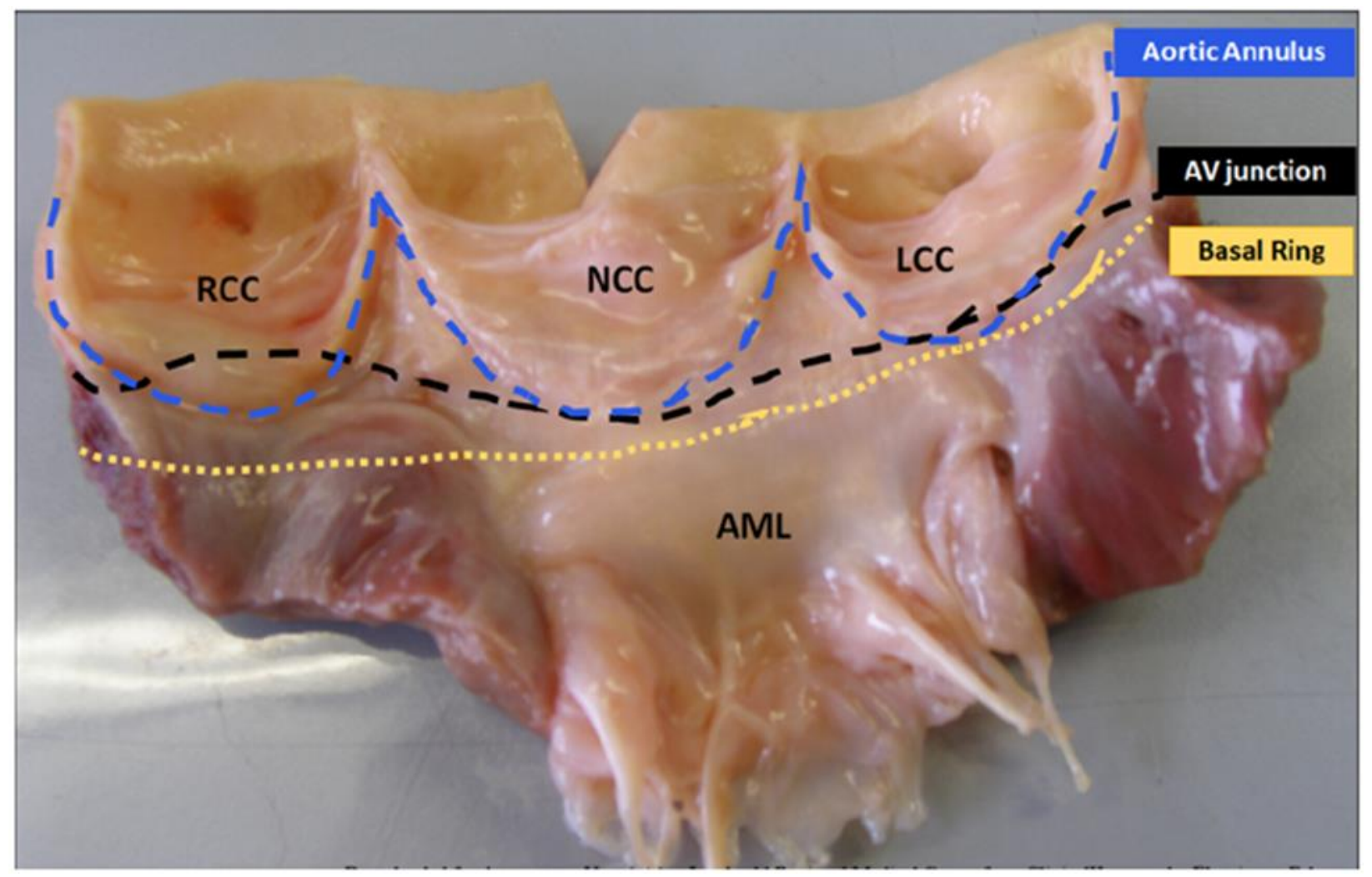

Figure 2: Opened aortic root (tricuspid aortic valve) showing the crown-shaped aortic annulus, the anatomic aortoventricular (AV) junction, and the basal ring. (AML, anterior mitral leaflet, LCC, leftcoronary cusp; NCC, noncoronary cusp; RCC, right coronary cusp.) From Ram D, Bouhout I, Karliova I, Schneider U, El-Hamamsy I, Schafers HJ. Concepts of bicuspid aortic valve repair: a review. Ann Thorac Surg 2020 Apr;109(4):999-1006.

Named after the Italian anatomist Antonio Valsalva, the SoV forms three distinct expanded portions of the aortic wall and is confined to the region bounded caudally by the valvular leaflets and cranially by the sinotubular ridge [12]. The function of the $\mathrm{SoV}$ is under investigation; however, it has been proposed that the area of the SoV creates flow turbulence, which may lead to a reduction in shear stress on the aortic leaflets and gradual valve closure, while supporting coronary flow [14].

\section{Surgical technique}

The most frequent mechanism for aortic regurgitation is dilation of the aortic root and ascending aorta. Therefore, we will begin by discussing the development of valve sparing root replacement. The aortic root remodeling procedure was initially described by Sarsam et al. In summary, in the aortic remodeling procedure, the aortic wall is excised to within approximately $3 \mathrm{~mm}$ of the leaflet attachments. Next, the coronary arteries are detached, and a Dacron graft is sized and sutured into the excised sinuses. Lastly, the coronary arteries are subsequently reimplanted [15].
A second option for accomplishing valve sparing aortic root replacement as originally described by David et al. [16]. is referred to as the reimplantation technique. This is a complex procedure, and a detailed description is beyond the scope of this review. In summary, the aneurysmal portion of the ascending aorta and SoV are excised, while leaving the aortic valve leaflets and portion of the arterial wall attached to the left ventricular outflow tract. Next, a carefully sized and constructed collagen-impregnated tubular Dacron graft is affixed proximally to the VAJ using pledgeted sutures placed, generally speaking, along the plane formed by the nadir of leaflet insertion [17]. The native valve is then implanted within the Dacron graft using a running suture. After valve reimplantation, identified leaflet pathology is corrected using techniques described below. The coronary arteries are subsequently re-attached, and the distal anastomosis between the graft and native aorta is performed (Figure 3). While technically more demanding, the reimplantation technique has the advantage of inherently stabilizing the aortic root at the level of the basal ring while the remodeling technique may be physiologically superior [18]. Both the reimplantaton and the remodeling 
technique have been successfully performed among patients with Marfan syndrome and aortic root aneurysm [19,20].

Although the initial results of these two procedures was promising, follow-up studies demonstrated recurrent AI [21]. It was initially unclear as to the cause or mechanism of recurrent aortic regurgitation until Schäfers et al. [22, 23] published findings that the valve-preserving aortic root replacement procedure itself induced aortic valve leaflet prolapse. Their institution were early adopters of combining cusp prolapse repair in conjunction with valve sparing root replacement. Furthermore, Schäfers et al. [24] went on to describe the effective height concept into AV repair, which has been nearly universally adopted among surgeons performing AV repair.

\section{The Effective Height Concept}

Prior to summarizing techniques used to accomplish aortic leaflet repair, an understanding of the effective height $(\mathrm{eH})$ concept is helpful. Various measurements are used to define aortic cusp geometry. These include dimensions of the SoV, annular dimensions, length of the free margin, and cusp height. Of the indices of cusp geometry, Schäfers et al. [24] determined that the distance between the basal plane (horizontal plane connecting the cusp nadirs) and central free margin is $8-10 \mathrm{~mm}$ in the normal aortic valve when measured in diastole; this measurement is termed the Effective Height (see Figures 4).

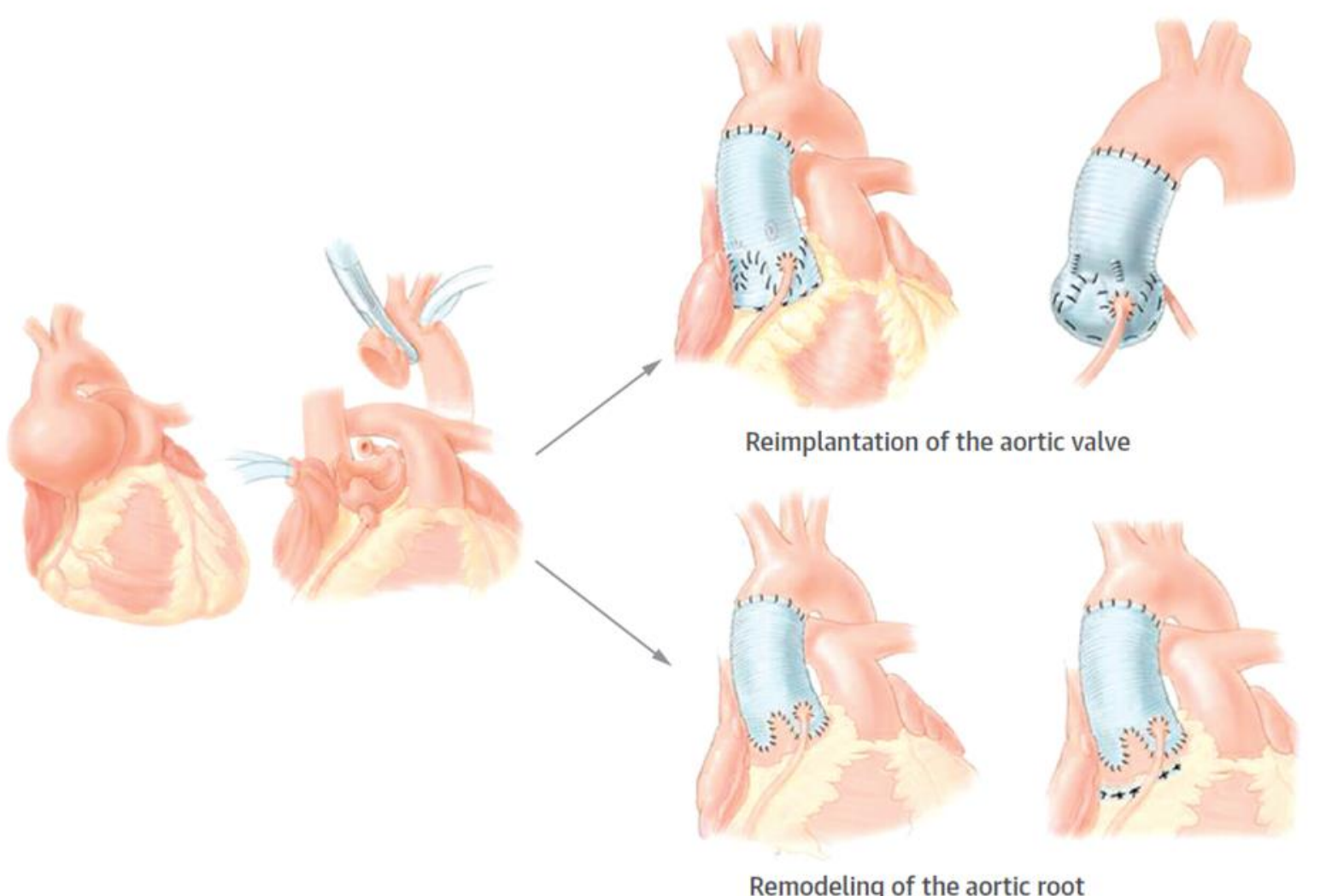

Figure 3: (David T. Aortic Valve Sparing in different aortic valve and aortic root conditions. J Am Coll Cardiol 2016; 68:654-64). 

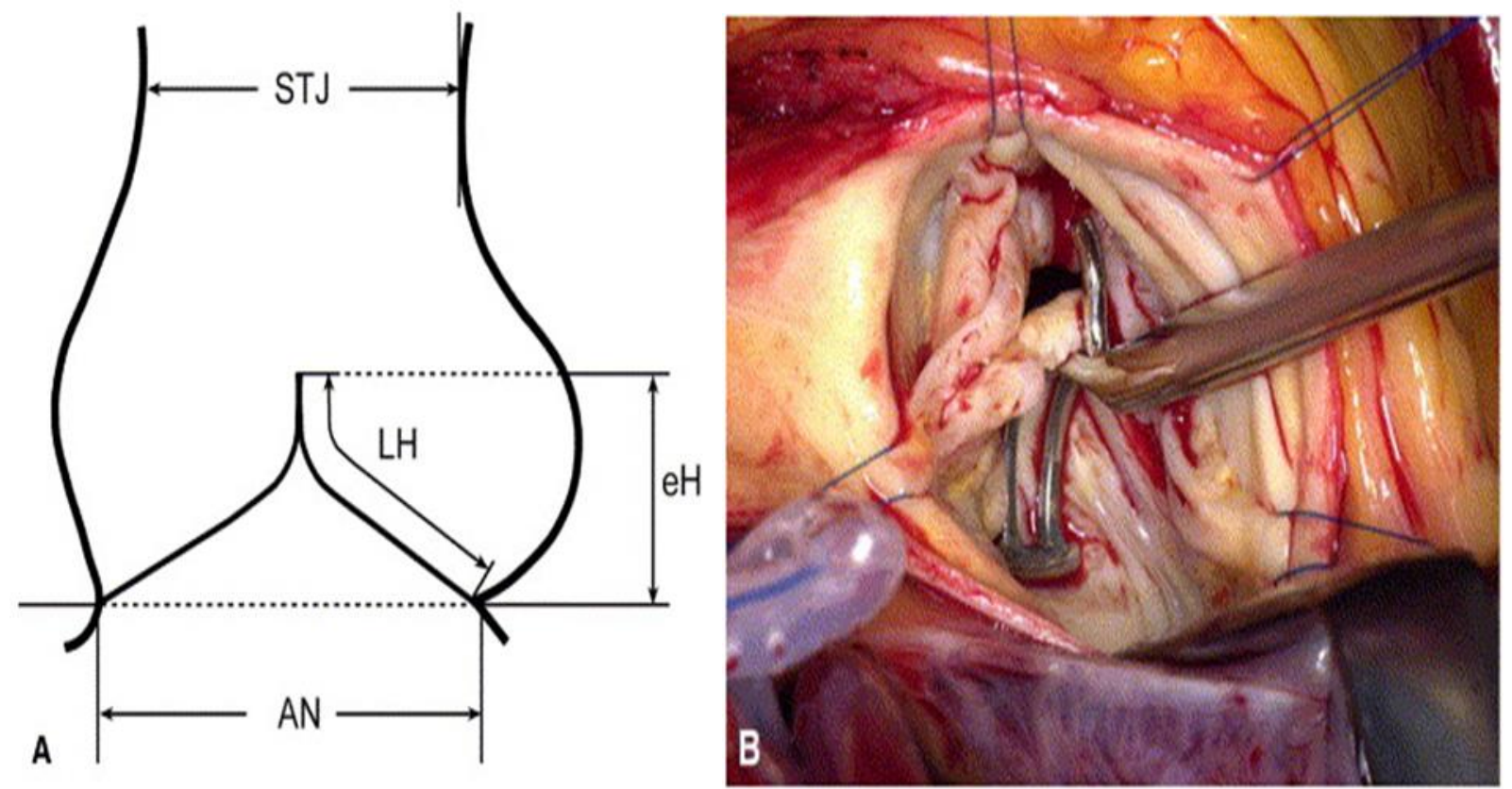

Figure 4: A. Effective height is the height difference between the central free margins and the aortic insertion lines. This can be measured intraoperatively with a caliper. STJ, Sinotubular junction; AN, annulus or aortoventricular junction; LH, leaflet or cusp height; eH, effective height.

B. Intraoperative photograph showing measurement of the effective height of the noncoronary cusp of a bicuspid aortic valve with the caliper. The caliper is placed such that the longer end rests on the lowest (i.e., central) point of the insertion line. The shorter end is pushed to the free margin, with the curve accommodating the margin.

(From Schafers H, Bierbach B, Aicher D. A new approach to the assessment of aortic cusp geometry. J Thorac Cardiovasc Surg 2006; 132(2):436438.)

It was further observed that, when the distance between the aortic insertion lines and central free margins is low (typical cutoff is less than $8 \mathrm{~mm}$ ) following aortic remodeling, there is increased risk for recurrent aortic regurgitation. However, efforts to measure the effective height intraoperatively proved initially difficult. To overcome this obstacle, a unique caliper was invented for the purpose of easily, accurately, and reproducibly determining effective height (MSS-1, Fehling Instruments, Karlstein, Germany). With the aid of this tool, repair techniques discussed below are performed with the intention of restoring an effective height of 9 to $10 \mathrm{~mm}$ (See Figure 4).

In summary, when performing aortic valve sparing root replacement, assessing and correcting aortic cusp prolapse using the concept of effective height, in conjunction with root remodeling or reimplantation, has refined and improved long-term results.

\section{Leaflet Repair}

Among patients with annular dilation as the primary mechanism causing $\mathrm{AI}$, approximately $88 \%$ have additional leaflet prolapse, which further contributes to the severity of AI [25]. A proposed explanation for the association between aortic dilation and leaflet prolapse is the hypothesis that progressive annular dilatation increases stress on valve leaflets. Consequently, over time, this may result in leaflet stretching and prolapse [25]. Thus, aortic leaflet prolapse may coexist with aortic root dilation (mixed etiology). In addition, aortic leaflet prolapse may occur as a consequence of aortic valve preserving root replacement, or isolated prolapse may be the sole cause of aortic valve insufficiency. A successful surgical outcome may thus require a combination repair of aortic root dilation and additional leaflet pathology (most commonly aortic leaflet prolapse by mean of distention of the free margin) [26,27]. We will now shift our attention to surgical methods used for leaflet pathology. We will discuss the most common methods used for aortic leaflet repair: central plication, leaflet resuspension, leaflet resection, and pericardial patch.

In the tricuspid aortic valve, surgical repair techniques aim to elevate the level of coaptation, and restore effective height. Although several techniques have been proposed, due to associated technical difficulties, many have largely been abandoned. A technique termed central plication has emerged as the primary modality used to repair a prolapsing aortic leaflet $[28,29]$. In this surgical method, simple 5-0 or 6-0 polypropylene plication sutures are placed within the thickened leaflet free-edge cord, with the intent of shortening the prolapsing leaflet free-edge length adjacent to the nodule of Arantius. An advantage of this technique is its relative simplicity when compared to alternative techniques and the ability to adjust cusp geometry in a stepwise manner [29]. One significant disadvantage of central plication is that this procedure is not feasible in circumstances involving significant leaflet calcification. 
The resuspension technique was developed, during which Gore-Tex sutures are passed in a running fashion over the entire length of the free margin. The free margin is shortened by applying tension on both GoreTex suture arms, which are subsequently locked. This technique may be a suitable option for closing aortic leaflet fenestrations and for reinforcing fragile free margins (30). Additionally, it is further possible to combine both plication and resuspension, as demonstrated by Kerchove et al. [31].

A technique which is particularly well suited in the presence of leaflet perforation or fenestration is the use of pericardial patch repair. However, several publications $[32,33]$ indicate that the use of pericardium for leaflet reconstruction may be associated with an increased rate of repair failure. In a retrospective review performed by Karliova et al., the authors observed that long-term stability following the use of pericardial patch repair is best suited for closure of fenestrations, followed by defect closure, and cusp augmentation. However, among bicuspid aortic valves, the use of pericardial patch was associated with particularly poor stability, regardless of technique [32]. The 10-year observed patch-related freedom from reoperation was only $78 \%$ among bicuspid AV repairs. Thus the durability after aortic repair using pericardium is dependent on both valve morphology and underlying cusp pathology.

In the presence of marked tissue redundancy $(>10 \mathrm{~mm})$ or dense fibrosis/calcification of the prolapsing cusp, triangular resection is a useful method. In this manner, a central resection of cusp tissue is created with the remaining tissue readapted using interrupted Prolene sutures [29]. If the resulting defect following triangular resection is too large to allow direct readaptation, then a pericardial patch is used for leaflet reconstruction.

\section{Surgical Repair of the bicuspid valve}

The anatomy of the bicuspid valve (BAV), as described by Sievers and Schmidtke (34), is divided into three general classifications. First, a Type 0 BAV consists of two symmetric aortic cusps without the presence of a central raphe (fused region of underdeveloped leaflets). The type 0 BAV occurs as the result of a complete failure in the development one commissure, resulting in two completely developed symmetric leaflets and commissures $[33,34]$. The mechanism of insufficiency is typically a result of excessive and redundant prolapsing cusp tissue. More common is the type $1 \mathrm{BAV}$, in which there are two fully developed commissures, one under-developed commissure, and a central raphe. The type 2 BAV occurs due to the full development of only one commissure, with two under-developed commissures, resulting in the presence of 2 raphe. The type 2 BAV may appear to be nearly tri-leaflet in configuration.

While the classification proposed by Sievers is the most widely adopted, there are recognized limitations with relevance to surgical planning for AV repair [35]. Thus, a novel repair-oriented classification scheme has recently been proposed by Kerchove et al. [36], which classifies bicuspid aortic valves in terms of the observed variability in degree of commissural orientation. In brief, the orientation of the two functional commissures has been observed to vary from 180 degrees (Kerchove Type A, symmetric such as described in a Sievers type 0) to 120-140 degrees (Kerchove Type C, very asymmetric, nearly tricuspid in configuration). Among asymmetric BAVs with a near tricuspid configuration, prolapse may preferentially involve the rudimentary right cusp.

The approach to repair of the BAV uses techniques similar to those described above. In the type 0 valve, with prolapse as the predominant mechanism of insufficiency, free margin plication or free margin resuspension with a goal for restoring effective height is the preferred approach. However, the repair technique is typically more complex in type 1 valves. If the raphe is relatively mobile and only mildly fibrosed, it may be preserved and shaved using a combination of a scalpel and scissors. However, if the raphe is found to be significantly calcified, then triangular resection is typically employed. Following triangular resection, the degree of remaining adequate cusp tissue is assessed. If deemed sufficient, then leaflets edges may be re-approximated using polypropylene sutures. However, in the absence of adequate tissue, the cusp may be restored with a bovine pericardial patch.

It is further observed that stability of BAV repair is affected by commissural orientation, as predicted by the Kerchove classification system described above. For example, one year freedom from reoperation was estimated to be less than $50 \%$ when preoperative commissural orientation was less than 160 degrees [37]. Schneider et al., demonstrated that plication of the fused sinus could effectively restore commissural orientation to greater than 160 degrees, and as a consequence could substantially improve outcomes [38] (See Figure 5). 

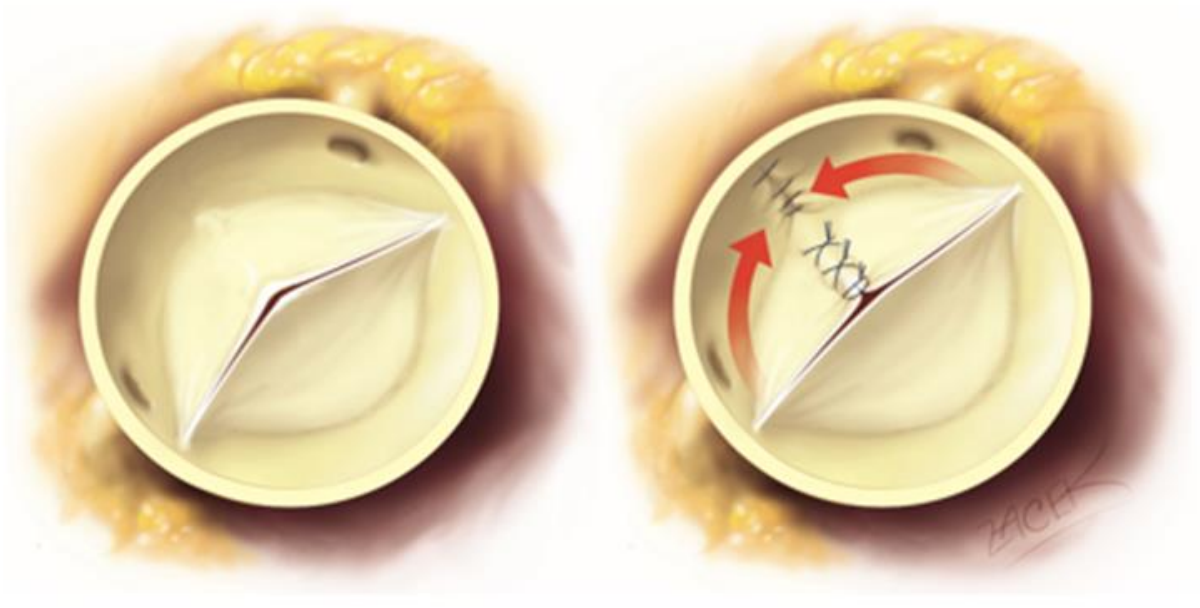

Figure 5: Repair of an asymmetric (Kerchove type B) bicuspid aortic valve. Correction of leaflet prolapse using plication sutures are depicted. In addition, plication of the fused sinus with the intent to restore commissural orientation is further illustrated.

From Ehrlich T, de Kerchove L, Vojacek J, et al. State-of-the art bicuspid aortic valve repair in 2020. [Published online ahead of print] Progress in Cardiovascular Diseases. doi: 10.1016/j.pcad.2020.04.010

In the presence of a dilated aortic root, the aortic root is typically replaced using the reimplantation technique. If the aortic root is not dilated, the aortic wall tissue is closely inspected. In circumstances where the tissue is found to be translucent or fragile, aortic root replacement may be indicated, even in the absence of aneurysm.

\section{Unicuspid valve}

The anatomy of the unicuspid aortic valve (UAV), as defined by Novaro and colleagues [39], consists of the presence of an eccentric valvular orifice with either a single (typically posterior) or complete absence of commissural attachment, and one aortic leaflet with or without visualization of a raphe. The UAV is generally considered to be a rare finding and is estimated to occur with a frequency of 0.02 percent. However, due to under-recognition, UAV may be more common than previously estimated. The visualization of an obtuse angle of valvular orifice opening at the site of the commissure and an eccentric orifice are useful echocardiographic features for identifying a UAV.

It is furthermore controversial as to whether the UAV is a distinct entity or represents a continuum of BAV disease. However, Noly et al [40] demonstrate several distinguishing features which are unique to UAV, such as relatively more frequent dilation at the level of the aortic annulus, which argues that these represent separate and distinct entities.

In the absence of significant stenosis or calcification, the regurgitant UAV may be successfully repaired. The bicuspidization procedure was described initially by Schafers et al. [41], during which the UAV is reconstructed into a bicuspid configuration through the creation of a second commissure. In this procedure the fused cusp is incised toward the anterior commissure, and subsequently detached from the aortic insertion. A second commissure is then formed using autologous pericardium which is sutured within the gap formed by the excised tissue. A recently published case series demonstrated excellent 26 month follow-up outcomes data for the bicuspidization procedure, for both stenotic and regurgitant $\mathrm{UAV}$, for a small population [42].

\section{Outcomes}

Currently, there are no head-to-head trials comparing surgical AV repair to that of valve replacement. However, several published reviews of AV repair are now available which provide contemporary outcomes for surgical aortic repair.

Results from a high-volume center over a 12-year period were published by Aicher et al. [43]. In their review of 640 AV repair cases over a 12 year period, the authors found a remarkably low overall incidence of valve-related complications. They report a hospital mortality rate of 3.4\% in the total patient cohort. A higher mortality risk was found among patients over the age of 70 (6.7\% mortality), among those who underwent concomitant coronary artery bypass graft (CABG) $(8.1 \%$ mortality rate), and emergency operations (7.8\%). However, after excluding these highrisk circumstances, mortality rate decreases to only $0.8 \%$ for cases involving isolated AV repair. Furthermore, freedom from reoperation at 5 and 10 years was $88 \%$ and $81 \%$ involving BAV, and $97 \%$ and $93 \%$ in tricuspid aortic valves $(\mathrm{p}=0.0013)$. For the 36 cases requiring reoperation, 13 out of 36 valves could be re-repaired, thus avoiding the need for valve replacement. Lastly, freedom from all valve-related complications (reoperation, thrombo-embolism, endocarditis, and hemorrhage) was $88 \%$ at 10 years (93\% in tricuspid valves vs. $80 \%$ involving BAV).

Boodhwani et al. [44] published their review of 122 non-emergent AV repair procedures involving strictly BAVs. The authors found that overall survival at 8 years was determined to be $97 \%+/-2 \%$. During an approximate 5-year follow-up period, seven reoperations occurred (majority for recurrent $\mathrm{AI}$ ), which corresponds to an overall freedom from reoperation of $94 \%+/-2 \%$ at 5 years and $83 \%+/-5 \%$ at 8 years. The 
authors further report four embolic events (one transient ischemic attack, three strokes) and no bleeding events over the same follow-up period.

Jasinski et al. [45] report their experience with AV repair for aortic regurgitation for 200 consecutive cases involving either tricuspid or bicuspid aortic valves over a 10 -year period. They found an overall survival at a mean follow up of 48 months was $94 \%+/-1.9 \%$. In their cohort, the overall 6-year freedom from reoperation was $90 \%$.

In a report authored by Schneider et al. (46), long-term outcomes among 852 patients with surgically repaired bicuspid aortic valve treated at Saarland University Medical Center between 1995 and December 2015 are published. The authors observe a cumulative incidence of reoperation of $12.3 \%$ at 10 years and $21.7 \%$ at 15 years. Furthermore, at 10 and 15 years, the cumulative incidence of aortic regurgitation grade II or higher was $12.3 \%$ and $17.1 \%$, respectively. There was a statistically significant associations of aortic valve calcification (HR, 4.34; 95\% confidence interval, 1.69-11.16; $\mathrm{P}=.002)$, and use of a pericardial patch for partial cusp replacement $(\mathrm{HR}, 4.00 ; 95 \%$ confidence interval, $1.65-9.66 ; \mathrm{P}=$ .002 ) with respect to time to reoperation.

Over the prior two decades several technical refinements have led to improved durability of BAV repair. Examples include: measuring effective height intra-operatively, the use of sinus plication with intent to favorably change commissural orientation, and the incorporation of suture annuloplasty. Consequently, a demonstrated freedom from reoperation among patients with bicuspid aortic valve of $87.5 \pm 2.8 \%$ and $80.1 \pm 2.6 \%$ at 10 and 15 years is reported in a contemporary patient cohort who underwent surgery between 2000 and 2018. The authors conclude that with current technique, more than $90 \%$ of regurgitant bicuspid aortic valves are repairable [47].

\section{Echocardiographic guided surgical planning}

A classification system was proposed by El Khoury at al. (48) for purposes of classifying the mechanism of aortic regurgitation. In this scheme, Type $1 \mathrm{AI}$ is described as AI due to abnormalities involving the aortic root. Type $1 \mathrm{AI}$ is further divided into several sub classifications. First, Type 1a is AI determined to be due to combined effacement of the STJ and dilation of the ascending aorta. On the other hand, Type 1b is valvular regurgitation due to dilation of the SoV and STJ. Type 1c is AI that results from dilation of the VAJ. Type $1 \mathrm{~d}$ is used to describe AI due to cusp perforation. Type $2 \mathrm{AI}$ is due to leaflet prolapse as a result of excessive cusp tissue or disruption of the commissure. Lastly, Type $3 \mathrm{AI}$ is due to leaflet restriction (as may be seen in cases involving bicuspid, degenerative, or rheumatic valve disease).

The above described classification system was shown by Boodwani et al. [49] to be relevant to preprocedural planning for surgical AV repair (Figure 6).

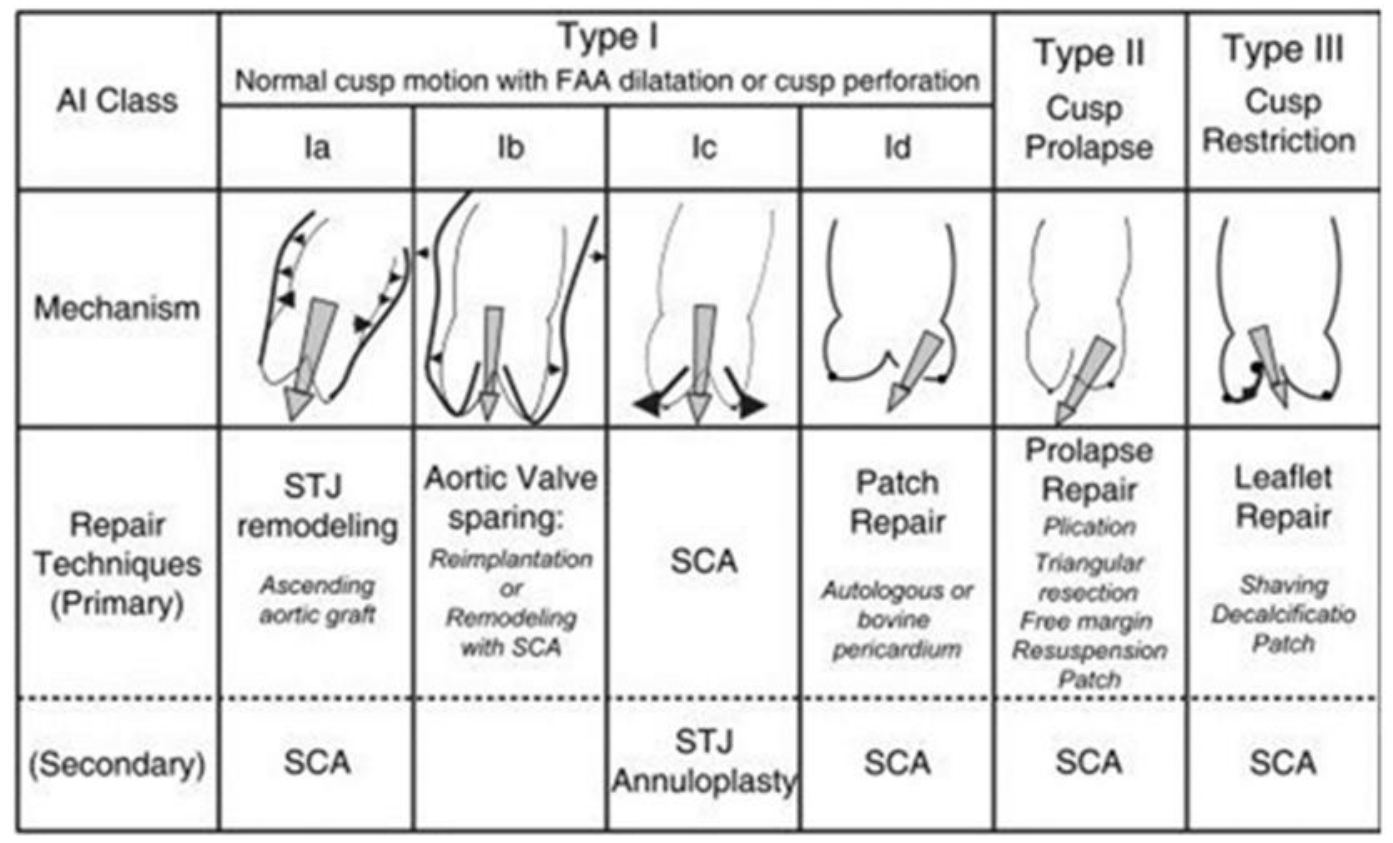

Figure 6: Repair-oriented functional classification of aortic insufficiency (AI) with description of disease mechanisms and repair techniques used. FAA, Functional aortic annulus; STJ, sinotubular junction; SCA, subcommissural annuloplasty (From Boodhwani M, Kerchove L, Glineur D, et al. Repair-oriented classification of aortic insufficiency: impact on surgical techniques and clinical outcomes. J Thorac Cardiovasc Surg 2009; 137(2):286-

In their retrospective review, the authors found that, by classifying the mechanism of AI by echocardiography during the preprocedural evaluation, they were subsequently able to predict surgical repair technique. Thus, it was determined that accurate preoperative assessment of the mechanism of AI can help guide and standardize surgical technique.
However, in spite of the findings demonstrated in this paper, adjunctive surgical techniques were necessary in as many as roughly $35 \%$ of patients on the basis of intraoperative findings, which were not anticipated during preoperative evaluation. Often, these adjunctive surgical techniques were required due to the finding of combined pathology (e.g., predominant 
Type 1c mechanism AI, with concomitant leaflet pathology, or induced leaflet prolapse following aortic root remodeling).

Recently, data have been published in regard to the use of 3-dimensional echocardiography in an attempt to improve preprocedural assessment of the mechanism of AI. First, 3-dimensional echocardiography helps to identify and classify the mechanism of AI into the classification system presented by Boodwani et al. This enables the fundamental framework with which operative planning may commence. However, 3-dimensional echocardiography further delineates morphology of the aortic valve leaflets, thereby improving preprocedural planning of intraoperative repair techniques by providing precise etiologic, morphologic, and functional assessments of aortic root pathology.

A detailed explanation regarding the 3-dimensional echocardiography protocol used to assess aortic cusp configuration is beyond the scope of this review. However, Hagendorff et al. [50] have published a proposed systematic approach with which to accomplish a detailed assessment of aortic cusp morphology pre- and post-surgical repair. In summary, multiplanar reconstruction allows adjustment of orthogonal imaging planes for optimal visualization of all AV coaptation lines [50,51]. After each coaptation point is identified, the effective height, geometric height, and coaptation length are determined. By determining the effective height preprocedurally, aortic cusp prolapse may be identified, thus helping to predict surgical strategies such as leaflet plication. Surgical strategy is further influenced by the potential findings of leaflet fenestrations or significant calcification of leaflet or aortic arch.

Transesophageal echocardiography is not only useful for preprocedural surgical planning and assessment but has been shown to identify patients at risk for developing recurrent severe AI following repair [52]. Several echocardiographic findings have been shown to predict recurrent aortic regurgitation. First, coaptation level below the aortic annulus is highly predictive for recurrence. Second, the absence of residual AI was shown to strongly favor long-term success. Coaptation length greater than $4 \mathrm{~mm}$ conferred a very low risk for AI recurrence. Last, Type 3 AI repair is associated with a high risk for recurrent AI. This is likely due to the frequent need to excise large areas of diseased tissue. As a consequence, the surgeon is frequently left with insufficient tissue to restore normal valve function.

\section{Conclusion}

There is growing awareness in regard to the limitations and shortcomings of surgical prosthetic valves. This is particularly true in the circumstance involving aortic valve surgery among our younger patient population. Consequently, there is a need for alternative surgical options for the treatment of aortic valve disease. Among properly selected patients, AV repair is one promising modality.

There has been a slow rate of adoption, particularly among institutions within the United States, for AV repair. One explanation for this is the historical lack of evidence and outcomes data for AV repair. However, new data show successful results for surgical AV repair, particularly in high-volume centers. Further surgical refinement has been accomplished with thoughtful preprocedural planning, with the aid of advanced echocardiographic imaging.
We hope that this review paper will encourage consideration for surgical AV repair among properly identified patients. In addition, we feel that AV repair may benefit from high-volume centers of excellence, with a Heart Team involving cardiologists with advanced imaging experience, who may provide valuable insight for purpose of surgical planning.

\section{References}

1) Glaser N, Persson M, Jackson V, Holzmann M, FrancoCereceda A, Sartipy U. (2019) Loss in life expectancy after surgical aortic valve replacement. J Am Coll Cardiol, 74:26-33.

2) Lansac E, Youssefi P, de Heer F, et al. (2019) Aortic Valve Surgery in Non-Elderly Patients: Insights Gained from AVIATOR. Semin Thorac Cardiovasc Surg, 31(4):643649.

3) Goldstone AB, Chiu P, Baiocchi M, et al. (2017) Mechanical or biologic prostheses for aortic-valve and mitral-valve replacement. N Engl J Med 377:1847-1857.

4) Chan V, Malas T, Lapierre H, et al. (2011) Reoperation of left heart valve bioprostheses according to age at implantation. Circulation 124(11) Suppl:75-80.

5) Buratto E, Shi WY, Wynne R, et al. (2018) Improved survival after the ross procedure compared with mechanical aortic valve replacement. J Am Coll Cardiol, 71:1297-1308.

6) Mazine A, El-Hamamsy I, Verma S, et al. (2018) Ross procedure in adults for cardiologists and cardiac surgeons. J Am Coll Cardiol, 72 (22):2761-2777.

7) de Meester C, Pasquet A, Gerber B, et al. (2014) Valve repair improves the outcome of surgery for chronic severe aortic regurgitation: A propensity score analysis. J Thorac Cardiovasc Surg, 148(5): 1913-1920.

8) Stamou S, Williams M, Gunn T, Hagberg R, Lobdell K, Kouchoukos N. (2015) Aortic root surgery in the United States: A report from the Society of Thoracic Surgeons database. J Thorac Cardiovasc Surg, 149(1):116-122

9) Charitos E, Sievers H. (2013) Anatomy of the aortic root: Implications for valve-sparing surgery. Ann Cardiothorac Surg, 2(1):53-56.

10) Anderson R. (2000) Clinical anatomy of the aortic root. Heart 84(6): 670-673.

11) Dyck M, Watremez C, Boodhwani M, Vanoverschelde J, Khoury G. (2010) Transesophageal echocardiographic evaluation during aortic valve repair surgery. Anesth Analg 111(1): 59-70.

12) Sutton J, Ho S, Anderson R. (1995) The forgotten interleaflet triangles: a review of the surgical anatomy of the aortic valve. Ann Thorac Surg, 59(2): 419-427.

13) Anderson H. (2003) Editorial: Understanding the Structure of the Unicuspid and Unicommissural Aortic Valve The Journal of Heart Valve Disease, 12:670-673

14) Katayama S, Umetani N, Sugiura S, Hisada T. (2008) The sinus of Valsalva relieves abnormal stress on aortic valve leaflets by facilitating smooth closure. J Thorac Cardiovasc Surg 136(6):1528-1535. 
15) Sarsam M, Yacoub M. (1993) Remodeling of the aortic valve annulus. J Thorac Cardiovasc Surg 105:435-438.

16) David T, Feindel C. (1992) An aortic valve-sparing operation for patients with aortic incompetence and aneurysm of the ascending aorta. J Thorac Cardiovasc Surg 103(4):617-622.

17) Kerchove L, Nezhad Z, Boodhwani M, et al. (2016) How to perform valve sparring reimplantation in a tricuspid aortic valve. Ann Cardiothorac Surg 2013; 2(1):105-112.

18) David T. (2016) Aortic Valve Sparing in different aortic valve and aortic root conditions. J Am Coll Cardiol, 68:654-64

19) David T.E., Armstrong S., Maganti M., Colman J., and Bradley T.J. (2009) Long-term results of aortic valvesparing operations in patients with Marfan syndrome. J Thorac Cardiovasc Surg, 138: pp. 859-864

20) David T. (2016) Aortic Valve Sparing in different aortic valve and aortic root conditions. J Am Coll Cardiol, 68:654-64

21) Oka T, Okita Y, Matsumori M, et al. (2011) Aortic regurgitation after valve-sparing aortic root replacement: modes of failure. Ann Thorac Surg 92(5):1639-1645.

22) Schafers H, Aicher D. (2013) Root remodeling for aortic root dilation. Ann Cardiothorac Surg 2(1): 113-116.

23) Schafers H, Aicher D, Langer F, Lausberg H. (2007) Preservation of the bicuspid aortic valve. Ann Thorac Surg 83(2): S740-745.

24) Schafers H, Bierbach B, Aicher D. (2006) A new approach to the assessment of aortic cusp geometry. J Thorac Cardiovasc Surg; 132(2):436-438.

25) Boodhwani M, Kerchove L, Glineur D, El Khoury G. (2010) A simple method for the quantification and correction of aortic cusp prolapse by means of free margin plication. J Thorac Cardiovasc Surg 139(4):1075-1077.

26) Langer F, Graeter T, Nikoloudakis N, Aicher D, Wendler O, Schafers H. (2001) Valve-preserving aortic replacement: does the additional repair of leaflet prolapse adversely affect the results? J Thorac Cardiovasc Surg 122:270-277.

27) Langer F, Aicher D, Kissinger A, et al. (2004) Aortic valve repair using a differentiated surgical strategy. Circulation, 110:Suppl 67-73.

28) Komiya T. (2015) Aortic valve repair update. Gen Thorac Cardiovasc Surg, 63(6): 309-319.

29) Aicher D, Kunihara T, Abou Issa O, Brittner B, Gräber S, Schäfers H. (2011) Valve configuration determines longterm results after repair of the bicuspid aortic valve. Circulation, 123(2): 178-185.

30) Lansac E, de Kerchove L. (2018) Aortic valve repair techniques: state of the art. Eur J Cardiothorac Surg, 53:1101-1107.

31) Kerchove L, Boodhwani M, Glineur D, et al. (2009) Cusp prolapse repair in trileaflet aortic valves: free margin plication and free margin resuspension techniques. Ann Thorac Surg, 88(2):455-461.
32) Aicher D, Langer F, Adam O, Tscholl D, Lausberg H, Schäfers H. (2007) Cusp repair in aortic valve reconstruction: does the technique affect stability? J Thorac Cardiovasc Surg 134(6): 1533-1539.

33) Karliova I, Schneider U, Ehrlich T, Schafers H. (2019) Results of pericardial patches in tricuspid and bicuspid aortic cusp repair. Ann Thorac Surg, [Epub ahead of print]

34) Sievers H, Schmidtke C. (2007) A classification system for the bicuspid aortic valve from 304 surgical specimens. J Thorac Cardiovasc Surg, 133(5):1226-1233.

35) Boodhwani M, Kerchove L, Glineur D, et al. (2010) Repair of regurgitant bicuspid aortic valves: a systematic approach. J Thorac Cardiovasc Surg 140(2): 276-284.

36) Kerchove L, Mastrobuoni S, Froede L, et al. (2019) Variability of repairable bicuspid aortic valve phenotypes: towards an anatomical and repair-oriented classification. Eur J Cardiothorac Surg, 56(2): 351-359.

37) Aicher $D$, Kunihara $T$, Abou Issa O, Brittner B, Graber $S$, Schafers HJ. (2011) Valve configuration determines longterm results after repair of the bicuspid aortic valve. Circulation, 123:178-185.

38) Schneider U, Schmeid W, Aicher D, Giebels C, Winter L, Schafers H. (2017) Sinus plication to improve valve configuration in bicuspid aortic valve repair-early results. Ann Thorac Surg, 103(2):580-585.

39) Novaro G, Mishra M, Griffin B, et al. (2003) Incidence and echocardiographic features of congenital unicuspid aortic valve in an adult population. J Heart Valve Dis Vol 12 No 6

40) Noly PE, basmadjian L, Bouhout I, et al. (2016) New insights into unicuspid aortic valve disease in adults: not just a subtype of bicuspid aortic valves. Canadian Journal of cardiology 32110-32116.

41) Shafers HJ, Aicher D, Riodionycheva S, et al. (2008) Bicuspidization of the unicuspid aortic valve: A new reconstructive approach. Ann Thorac Surg 85:2012-2018.

42) Kolesar A, Toporcer T, Bajmoczi M, et al. (2018) Aortic valve repair of a stenotic unicuspid aortic valve in young patients. Ann Thorac Surg 105:1351-1356.

43) Aicher D, Fries R, Rodionycheva S, Schmidt K, Langer F, Schäfers H. (2010) Aortic valve repair leads to a low incidence of valve-related complications. Eur $\mathbf{J}$ Cardiothorac Surg 37(1): 127-132.

44) Boodhwani M, Kerchove L, Glineur D, et al. (2010) Repair of regurgitant bicuspid aortic valves: a systematic approach. J Thorac Cardiovasc Surg, 140(2): 276-284.

45) Jasinski M, Gocol R, Malinowski M, Hudziak D, Duraj P, Deja M. (2015) Predictors of early and medium-term outcome of 200 consecutive aortic valve and root repairs. J Thorac Cardiovasc Surg 149(1): 123-129.

46) Schneider U, Feldner S, Hofmann C, et al. (2017) Two decades of experience with root remodeling and valve repair for bicuspid aortic valves. J Thorac Cardiovasc Surg 153(4): S65-71.

47) Miyahara S, Schneider U, Morgenthaler L, Schafers H. (2019) (Almost) All nonstenotic bicuspid aortic valves 
should be preserved or repaired. Semin Thorac Cardiovasc Surg 31(4):656-660.

48) El Khoury G, Glineur D, Rubay J, et al. (2005) Functional classification of aortic root/valve abnormalities and their correlation with etiologies and surgical procedures. Curr Opin Cardiol, 20(2):115-121.

49) Boodhwani M, Kerchove L, Glineur D, et al. (2009) Repair-oriented classification of aortic insufficiency: impact on surgical techniques and clinical outcomes. J Thorac Cardiovasc Surg, 137(2):286-294.

50) Hagendorff A, Evangelista A, Fehske W, Schafers H. (2019) Improvement in the assessment of aortic valve and aortic aneurysm repair by 3-dimensional echocardiography. JACC Cardiovasc Imaging, 12(11 Pt 1):2225-2244.

51) Nijs J, Gelsomino S, Kietselaer B, et al. (2014) 3D-echo in preoperative assessment of aortic cusps effective height. World J Cardiol, 6(7):689-691.

52) le Polain de Waroux J, Pouleur A, Robert A, et al. (2009) "Mechanisms of recurrent aortic regurgitation after aortic valve repair: predictive value of intraoperative transesophageal echocardiography." JACC Cardiovasc Imaging, 2(8):931-939.
This work is licensed under Creative Commons Attribution 4.0 License

To Submit Your Article Click Here: Submit Article

DOI: $10.31579 / 2641-0419 / 073$
Ready to submit your research? Choose Auctores and benefit from:

$$
\begin{aligned}
& \text { * fast, convenient online submission } \\
& \text { rigorous peer review by experienced research in your field } \\
& \text { * rapid publication on acceptance } \\
& \text { * uuthors retain copyrights } \\
& \text { * imique DOI for all articles } \\
& \text { immediate, unrestricted online access }
\end{aligned}
$$

At Auctores, research is always in progress.

Learn more www.auctoresonline.org/journals/clinical-cardiology-andcardiovascular-interventions 